\begin{abstract}
MS03 O1
Structural basis of the iron storage and delivery functions of frataxin $\mathrm{S}$. Al-Karadaghi ${ }^{1}$, T. Karlberg ${ }^{1}$, U. Schagerlof ${ }^{1}$, O. Gakh ${ }^{2}$, S. Park ${ }^{2}$, U. Ryde ${ }^{3}$, M. Lindahl ${ }^{1}$, K. Leath $^{4}$,E.Garman ${ }^{4}$, G. Isaya ${ }^{2}{ }^{1,3}$-Departments of Molecular Biophysics \& Theoretical Chemistry, Lund University, Sweden ${ }^{2}$-Departments of Pediatric \& Adolescent Medicine and Biochemistry \& Molecular Biology, Mayo Clinic College of Medicine, Rochester, USA, ${ }^{4}$-Department of Biochemistry, University of Oxford, UK.

E-mail: salam.al-karadaghi@mbfys.lu.se
\end{abstract}

Keywords: catalytic mechanisms, iron storage, iron delivery

Frataxin performs key functions in iron delivery and detoxification via a currently unknown mechanism. The crystal structure of the iron-free and iron-loaded frataxin trimer and a single particle electron microscopic reconstruction of a 24-subunit iron-free and iron-loaded oligomers provide a basis for understanding the mechanisms of frataxin self-assembly in oligomeric particles, iron acquisition, detoxification and storage. They also provide an insight into the interplay between frataxin and other proteins, to which iron is delivered. The structure of the trimer suggests that a gated mechanism controls iron delivery to different targets and iron storage, a combination of critical biological roles not found in other known iron-binding proteins. Since the trimer seems to exhibit structural details consistent with both such roles, we propose that it represents the primary functional unit of frataxin. Moreover, the trimer structure suggests that mutations found in patients with Friedreich's ataxia may destabilize trimer formation. Thus, compounds that would stabilize the frataxin trimer may provide a means to maximize the frataxin function in individuals affected by Friedreich's ataxia. An additional remarkable feature of frataxin oligomers to be discussed in this presentation is the striking functional similarities of frataxin particles to the evolutionary unrelated ferritin superfamily of iron storage proteins.

MS03 O2

Insights into sucrose isomerization from Sucrose Isomerase crystal structures Nushin Aghajari, CNRS/University Lyon 1, Lyon, France

E-mail: n.aghajari@ibcp.fr

Keywords: Sucrose isomerase, specificity, enzymesubstrate complex

Trehalulose ( $\alpha$-D-glucosylpyranosyl-1,1-D-fructofuranose) and isomaltulose ( $\alpha$-D-glucosylpyranosyl-1,6-Dfructofuranose) are structural isomers of sucrose ( $\alpha$-Dglucosylpyranosyl-1,2- $\beta$-D-fructofuranoside) which have similar taste profiles and very similar physical and organoleptic properties to sucrose. Moreover they are noncariogenic and their absorption reduces the rate with which monosaccharides and insulin are released into the bloodstream, why they can be applied in diabetic and sports foods and drinks. Considering also that no side effects have been yet reported, these compounds could be ideal sucrose substitutes. Furthermore, the reducing property of isomaltulose makes it an attractive industrial precursor for the manufacturing of biosurfactants and biocompatible polymers.

We have recently cloned, purified, crystallized [1], [2] and solved the structures of the sucrose isomerases, MutB, from Pseudomonas mesoacidophila MX-45, which mainly produces trehalulose, and of SmuA from Protaminobacter rubrum which mainly produces isomaltulose. Since sucrose is an inexpensive and readily available D-glucose donor, the industrial potential of these enzymes for synthesis of trehalulose and/or isomaltulose is large. For optimal utilization of the enzymes, targeting controlled synthesis of these functional isomers starting from sucrose, it is necessary to minimize the side reactions. This requires a thorough analysis of substrate binding modes and of the specificity-determining sites in the 3Dstructures.

We have investigated the catalytic mechanism and the specificities of MutB by combining mutagenesis and structural approaches, and report the first structure of a trehalulose synthase, and the first enzyme-substrate complex structures for a sucrose isomerase.

[1] Ravaud S.; Watzlawick H.; Mattes R.; Haser R.; Aghajari N. Acta Cryst F 2005, 61, 100.

[2] Ravaud S.; Watzlawick H.; Haser R.; Mattes R.; Aghajari N. Acta Cryst F 2006, 62, 74 .

\section{MS03 O3}

Crystallographic and in crystallo Raman spectroscopic studies of iron-peroxide intermediates in superoxide reductase Gergely Katona ${ }^{\text {a }}$, Philippe Carpentier ${ }^{\mathrm{a}}$, Vincent Nivière $^{c}$, Patricia Amara ${ }^{\mathrm{a}}$, Virgile Adam ${ }^{\mathrm{b}}$, Jeremy Ohana ${ }^{\mathrm{a}}$, Nikolay Tsanov ${ }^{\mathrm{a}}$, \& Dominique Bourgeois ${ }^{\mathrm{a}, \mathrm{b}}{ }^{\mathrm{a}} I B S$, Institut de Biologie Structurale, Grenoble, France. ${ }^{b}$ European Synchrotron Radiation Facility, Grenoble, France. ${ }^{c}$ Laboratoire de Chimie et Biologie des Métaux, iRTSVCEA/CNRS/Université J. Fourier, Grenoble, France.

E-mail: gergely.katona@ibs.fr

Keywords: Raman spectroscopy, iron-peroxide intermediate, intermediate trapping

Iron-peroxide intermediates are involved in the catalytic cycle of many iron-containing enzymes. Such intermediates were also identified in superoxide reductase (SOR) a non-heme mononuclear iron-enzyme that neutralizes superoxide radicals [1-3]. By diffusing hydrogen peroxide into SOR crystals, we trapped iron(III)(hydro)peroxo species. X-ray diffraction data and nonresonant Raman spectra recorded in crystallo revealed "end-on" iron-(hydro)peroxo configurations. Raman spectroscopy also monitored the influence of X-ray radiation on the trapped intermedate. The ${ }^{18} \mathrm{O}$ isotopic shifts of the iron-peroxide specific vibration bands confirmed the direct involvement of hydrogen peroxide in the formation of the intermediate.

The open SOR active site promotes the formation of transient hydrogen bond networks, which presumably assist the cleavage of the $\mathrm{Fe}-\mathrm{O}$ bond in order to release the reaction product, hydrogen peroxide. Multiple observations of the SOR active sites in the asymmetric unit allowed us to propose a structural mechanism which directs the processing of reactive oxygen species without producing highly reactive iron (IV) oxo intermediates. 
Density functional theory studies described the protonation state and the hydrogen bond network of the iron-peroxide intermediates in greater detail.

[1] - M. D. Clay et a1., J. Am. Chem. Soc. 2002, 124, 788.

[2] - J. P. Emerson et al., Biochemistry 2002, 41, 4348.

[3] - C. Mathe et al., J. Am. Chem. Soc. 2002, 124, 4966.

\section{MS03 O4}

A novel $\alpha-N$-acetylgalactosaminidase family with a $\mathrm{NAD}^{+}$dependent catalytic mechanism suitable for enzymatic removal of blood group $A$ antigens

Gerlind Sulzenbacher ${ }^{\mathrm{a}}$, Qiyong Peter Liu, Eric P. Bennett $^{\mathrm{c}}$, Martin L. Olsson ${ }^{\mathrm{d}, \mathrm{e}}$, Jean Spence ${ }^{\mathrm{b}}$, Ed Nudelman $^{\mathrm{b}}$, Steve Levery ${ }^{\mathrm{f}}$, Yves Bourne, Bernard Henrissat $^{\mathrm{a}}$ and Henrik Clausen ${ }^{\mathrm{c}, \mathrm{g}}$. " ${ }^{a}$ Architecture et Fonction des Macromolécules Biologiques, UMR6098, CNRS, Universités Aix-Marseille I \& II, Case 932, 163 Avenue de Luminy, 13288 Marseille Cedex 9, France. ${ }^{b}$ ZymeQuest Inc, 100 Cummings Center Suite 436H, Beverly, MA 01920. 'Departments of Cellular and Molecular Medicine and Oral Diagnotics, University of Copenhagen, Blegdamsvej 3, DK-2200 Copenhagen N, Denmark. ${ }^{d}$ Division of Hematology and Transfusion Medicine, Department of Laboratory Medicine, Lund University and University Hospital Blood Center, SE22185, Lund, Sweden. ${ }^{e}$ Department of Pathology, Beth Israel Deaconess Medical Center and Harvard Medical School, Boston, MA 02215, USA. ${ }^{f}$ Department of Chemistry, University of New Hampshire, Durham, NH 03824. ${ }^{g}$ Hematology Division Brigham \& Women's Hospital and Harvard Medical School, Boston, MA 02115, USA.

Keywords: Blood group conversion; glycosidase mechanism; convergent evolution

Enzymatic removal of blood group A and B antigens to develop universal red blood cells was a pioneering vision originally proposed 25 years ago. Processes for group B red cells have previously been developed and feasibility of the approach demonstrated in clinical trials. A major obstacle has been the lack of $\alpha-N$ acetylgalactosaminidases for efficient removal of A antigens. We describe a novel family of prokaryotic $\alpha-N$ acetylgalactosaminidases with a unique $\mathrm{NAD}^{+}$-dependent catalytic mechanism deduced from x-ray crystallography [1]. Using a recombinant protein from this gene family, an efficient process for enzymatic removal of A antigens from blood group A red cells was developed. The digestion process is performed at neutral $\mathrm{pH}$ within $60 \mathrm{~min}$ and uses $50 \mathrm{mg}$ enzyme/unit blood. This process holds promise for finally achieving the goal of universal red blood cells with significant improvements in safety and cost-effectiveness for transfusion medicine. The properties of family GH109 $\alpha$ - $N$-acetylgalactosaminidases with neutral $\mathrm{pH}$ optimum and favorable substrate specificity for branched blood group A structures are unique and distinctly different from those of other families containing $\alpha-N$-acetylgalactosaminidases. The three-dimensional structure of $\alpha-N$-acetylgalactosaminidase is unique amongst glycosidases structurally elucidated so far, but exhibits similarity to oxidoreductases, and its proposed catalytic mechanism involving $\mathrm{NAD}^{+}$is only the second example of a $\mathrm{NAD}^{+}$based glycolytic mechanism. Thus, the novel glycosidase family GH109 considerably expands our knowledge of the diversity of glycosidase folds and mechanisms.

[1] Liu, Q.P. et al. Nat. Biotechnol. 25, 454-464 (2007).

MS03 O5

Restriction endonucleases that resemble a component of the bacterial DNA repair machinery Matthias Bochtler, ${ }^{\mathrm{a}, \mathrm{b}}$, Monika Sokolowska ${ }^{\mathrm{a}, \mathrm{b}}$, Magdalena KausDrobek $^{\mathrm{a}, \mathrm{b}}$, Honorata Czapinska ${ }^{\mathrm{a}, \mathrm{b}}$, Roman Szczepanowski ${ }^{\mathrm{a}, \mathrm{b}}$, Gintautas Tamulaitis ${ }^{\mathrm{c}}$, Virginijus Siksnys ${ }^{c}$, International Institute of Molecular and Cell Biology, Trojdena 4, 02-109 Warsaw, Poland ${ }^{\mathrm{b}} \mathrm{MPI}-\mathrm{CBG}$, Pfotenhauerstr. 108, 01309 Dresden, Germany ${ }^{\mathrm{c}}$ Institute of Biotechnology, Graiciuno 8, LT-02241, Vilius, Lithuania E-mail: MBochtler@iimcb.gov.pl

\section{Keywords: DNA; restriction endonuclease; repair}

It has long been known that most type II restriction endonucleases share a conserved core fold and similar active sites. The same core folding motif is also present in the MutH protein, a component of the bacterial DNA mismatch repair machinery. In contrast to type II restriction endonucleases, which assemble into functional dimers to make a double strand break, MutH (/GATC, "/" indicates the cleavage site) is a monomer and nicks hemimethylated DNA. We have shown that restriction endonucleases $\mathrm{BcnI}(\mathrm{CC} / \mathrm{SGG}, \mathrm{S}$ stands for $\mathrm{C}$ or $\mathrm{G})$ and MvaI (CC/WGG, W stands for A or T), which catalyze double-strand breaks in pseudopalindromic DNA, are also monomers that recognize their pseudosymmetric targets asymmetrically. The crystallographic results demonstrate that $\mathrm{BcnI}$ and MvaI resemble MutH more closely than other restriction endonucleases and establish a close link between some restriction enzymes and the DNA repair machinery [1], [2].

[1] Kaus-Drobek M., Czapinska H., Sokolowska M., Tamulaitis G., Szczepanowski R., Urbanke C., Siksnys V. and Bochtler M. Nucleic Acids Res 2007, epub ahead of print

[2] Sokolowska M., Kaus-Drobek M., Czapinska H., Tamulaitis G., Szczepanowski R., Urbanke C., Siksnys, V. and Bochtler M. J. Mol. Biol. 2007, in press 